\title{
COMMUTING AND TOPOLOGICAL DENSITIES
}

\section{AND LIFTINGS}

BY

TREVOR J. MCMINN

\begin{abstract}
For fairly general conditions on a measure space, a group of bijections on the space and a topology on the space, densities and lifttings commuting with members of the group and with topologies finer than the given topology are obtained.
\end{abstract}

Introduction. Our objectives here are twofold, in fairly general settings to obtain for a measure (1) densities and liftings which commute with members of a group of bijections and (2) densities and liftings whose topologies are finer than a given topology. The space we deal with usually has finite measure or can be suitably partitioned into measurable subsets of finite measure. In getting a commuting density for a countable group we do not require the group to be amenable. Our condition for passage from a commuting density to a commuting lifting is a trifle weaker than a previously known condition of no nontrivial fixed points. With this and a kind of uniformity condition satisfied in connection with an invariant Borel family, a commutative lifting is obtained for a measure whose measurable sets each lie within a set of measure zero of a member of this family. Topologically, we get slightly better than the existence of a lifting whose topology is finer than a given metrizable topology with nonempty members included among the measurable sets of positive measure. Nowhere do we make any assumptions of local compactness.

The main avenue to these ends is the new proof of D. Maharam's [13] fundamental existence theorem for a lifting recently published by M. Sion [17]. Sion's simple direct proof relies only on basic notions of Carathéodory measures on abstract spaces and emphasizes connections with differentiation somewhat in the spirit of J. von Neumann [16] working with Lebesgue measure on the line in answer to the first question of this kind raised by $A$. Haar. We have modified Sion's approach in some respects, but in many details and

Received by the editors March 11, 1974 and, in revised form, October 12, 1974. AMS (MOS) subject classifications (1970). Primary 28A50, 46G15, 28A15; Secondary 28A10, 28A05, 04A15. 
and in the development of the general framework as embodied in our elaborations the approach is very much the same. We have broadened his definition of a differentiation system in a minor way to give us the same results but at the same time make the hypotheses of the lifting and other theorems more often satisfied in applications. More importantly, in an inductive argument, we have found it possible to go all of the way with densities before passing to the final lifting. This gives us the freedom we need to get further results when a group structure is grafted on. Thus we can sometimes still get a commuting density in situations which preclude a commuting lifting. Also in the metric case the construction of a lifting with finer topology is facilitated.

Good guides to the literature of this subject are the book by $A$. and C. Ionescu-Tulcea [7] and the survey paper by C. Ionescu-Tulcea [11]. Most of the known results on commuting and strong densities and liftings are due to these authors. More recent results can be found in [1], [2], [3], [6], [8], [9], [10], [14], [19]. All of the measure-theoretic and topological preliminaries we need can be found, among many other places, in [18].

1. Basic definitions and notations. 1. $S \sim A=\{x \in S: x \notin A\}$. When $S$ is understood we may just write $\sim A$ for $S \sim A$. Thus $S \sim A=S \cap \sim A$.

2. $\sigma F=\bigcup_{a \in F} \alpha ; \pi F=\bigcap_{a \in F} a$; sb $A=\{a ; \alpha \subset A\} ; \operatorname{sp} A=\{a ; A \subset a\}$.

3. $A \sim \beta=\{a \sim b: a \in A$ and $b \in B\} ; A \cap \cap B=\{a \cap b: a \in A$ and $b \in B\}$.

4. $\sigma^{\prime} F, \sigma^{\prime \prime} F, \sigma^{-} F$ are the families of all, respectively, finite, countable and arbitrary unions of members of $F ; \pi^{\prime} F, \pi^{\prime \prime} F, \pi^{-} F$ are the families of all, respectively, finite, countable and arbitrary intersections of members of $F$ intersected with $\sigma F$.

5. $F$ is a field on $S$ if and only if $\sigma F=S$ and for each $\alpha, \beta \in F, S \sim$ $\alpha \in F$ and $\alpha \cup \beta \in F ; F$ is a Borel field on $S$ if and only if $\sigma F=S$ and for each $\alpha \in F$ and countable $H \subset F, S \sim \alpha \in F$ and $\sigma H \in F$;

$$
\text { field } \begin{aligned}
F & =\text { the field on } \sigma F \text { generated by } F \\
& =\sigma^{\prime} \pi^{\prime}(F \cup\{\sigma F \sim \alpha: \alpha \in F\}) \\
& =\text { the smallest field on } \sigma F \text { including } F \\
& =\pi\{G: F \subset G \text { is a field on } \sigma F\} ;
\end{aligned}
$$

Borel $F=$ the Borel field on $\sigma F$ generated by $F$

$=$ the smallest Borel field on $\sigma F$ including $F$

$=\pi\{G: F \subset G$ is a Borel field on $\sigma F\}$.

6. $\operatorname{dmn} f=$ the domain of $f$; rng $f=$ the range of $f ; f \mid A=f \cap\{(x, y)$ : $x \in A\} ; f \circ g=$ the function such that $(f \circ g)(x)=f(g(x)) ; \chi A, S=\{(x, y): x \in A$ 
implies $y=1$ and $x \in S \sim A$ implies $y=0\}$.

7. $\omega$ is the set of natural numbers, $0,1,2, \ldots$; we assume that each natural number is the set of all such less than it; thus the natural number 0 is also the empty set.

8. $\mu$ is a Caratheodory measure on $S$ if and only if $\mu$ is such a function on sb $S$ that, for each $A \subset S$ and countable $H$ with $A \subset \sigma H \subset S, 0 \leq \mu(A) \leq$ $\Sigma_{a \in H^{\prime}} \mu(\alpha) \leq \infty$.

$\Pi_{\mu}=$ the family of $\mu$-measurable sets

$=\{A \in \operatorname{dmn} \mu:$ for each $T \in \operatorname{dmn} \mu, \mu(T)=\mu(T \cap A)+\mu(T \sim A)\} ;$

9. $\mathbb{M}_{\mu}^{+}=\left\{A \in \mathbb{M}_{\mu}: 0<\mu(A)\right\} \cup\{0\}$;

$\mathbb{M}_{\mu}^{0}=\left\{A \in \mathbb{M}_{\mu}: \mu(A)=0\right\}$.

(Note that $\mathbb{M}_{\mu}^{+} \cap \mathbb{M}_{\mu}^{0}=\{0\}$.)

10. $A \equiv{ }_{\mu} B$ if and only if $\mu((A \sim B) \cup(B \sim A))=0$.

11. Borelnil ${ }_{\mu} F=\operatorname{Borel}\left(F \cup \pi_{\mu}^{0}\right)$.

$\mu / F=$ the Carathéodory measure generated by $\mu \mid F ;$

12. $\mu / F(A)=\inf \left\{\sum_{a \in H} \mu(\alpha): F \supset H\right.$ is countable and $\left.A \subset \sigma H\right\}$.

13. $F$ is $\mu$-complete if and only if for each $A \in F$ and $A^{\prime} \in \mathrm{dmn} \mu$ with $A^{\prime} \equiv{ }_{\mu} A, A^{\prime} \in F$.

14. $H$ is $G$-invariant if and only if for each $A \in H$ and $t \in G, t[A] \in H$; $\rho$ is $G$-commutative if and only if for each $A \in \operatorname{dmn} \rho$ and $t \in G, t[A] \in \operatorname{dmn} \rho$ and $\rho(t[A])=t[\rho(A)]$; we shall write $t \cdot z$ for both $t(z)$ and $t[z]$.

15. $B_{\mu}(S)=$ the family of all $\mu$-essentially bounded $\mu$-measurable extended real-valued functions on $S$.

16. $B_{\mu}(S)$ is $G$-invariant if and only if for each $f \in B_{\mu}(S)$ and $t \in G$, $f \circ t \in B_{\mu}(S) ; L$ is $G$-commutative if and only if for each $f \in B_{\mu}(S)$ and $t \in G$, $f \circ t \in B_{\mu}(S)$ and $L(f \circ t)=L(f) \circ t$.

17. $f \equiv_{\mu} g$ if and only if $\mu\{x \in \sigma \mathrm{dmn} \mu: f(x) \neq g(x)\}=0$.

2. Assumptions. Throughout what follows we shall assume that $S$ is a fixed set, $\mu$ is a fixed Carathéodory measure on $S$ with $0<\mu(S), G$ is a fixed group of one-to-one functions on $S$ onto $S$ (bijections), $e$ is the identity of $G, \gamma$ is the cardinality of $G, g$ is a one-to-one function on $\gamma$ onto $G$ with $g_{0}=e$ and $\mathcal{T}$ is a fixed topology on $S$. Frequently, the mention of ' $S$ ', ' $\mu$ ', ' $G$ ' and ' $T$ ' in formulas will be suppressed. 
3. Densities and liftings.

1. Definition. $\rho$ is a density on $\mathbb{B}$ if and only if $0, s \in \mathcal{B} \subset \mathbb{M}$ and $\rho$ is such a function on $\mathcal{B}$ to $\mathbb{M}^{+}$that, for each $A, B \in \mathcal{B}$,

.1. $\rho(A) \equiv A$,

.2. $A \equiv B$ implies $\rho(A)=\rho(B)$,

3. $A \cap B \in \mathcal{B}$,

.4. $\rho(A \cap B)=\rho(A) \cap \rho(B)$,

.5. $\rho(S)=S$.

It immediately follows that for each $A, B \in \mathfrak{B}$,

.6. $A \in \pi^{0}$ implies $\rho(A)=0$,

.7. $\sim A \in \mathscr{B}$ implies $\rho(A) \subset \sim \rho(\sim A)$,

.8. $A \subset B$ implies $\rho(A) \subset \rho(B)$,

.9. $\rho(A) \sim \rho(B) \in \pi^{+}$,

.10. $A \subset \rho(A), \sim A \in \mathcal{B}$ and $\sim A \subset \rho(\sim A)$ imply $A \in \mathrm{mg} \rho$.

2. Definition. $\rho^{\prime}<<\rho$ if and only if $\rho^{\prime}, \rho$ are such densities with complete field domains that

.1. $\mathrm{dmn} \rho^{\prime} \subset \mathrm{dmn} \rho$,

.2. for each $A^{\prime} \in \operatorname{dmn} \rho^{\prime}, \rho^{\prime}\left(A^{\prime}\right) \subset \rho\left(A^{\prime}\right)$.

It immediately follows that

.3. if $\rho^{\prime}<<\rho$ and $A^{\prime} \in \operatorname{dmn} \rho^{\prime}$ and $A \in \operatorname{dmn} \rho$, then $\rho^{\prime}\left(A^{\prime}\right) \equiv \rho\left(A^{\prime}\right)$ and $\rho^{\prime}\left(A^{\prime}\right) \cap \rho(A) \in \mathbb{M}^{+}$,

.4. $<<$ is reflexive, antisymmetric and transitive,

.5. if $\rho^{\prime}, \rho$ are such densities with complete field domains that $\rho^{\prime} \subset \rho$, then $\rho^{\prime}<<\rho$,

.6. if $K$ is a family of densities totally ordered by $<<$ and if, for each $A \in \bigcup_{\rho^{\prime} \in X} \operatorname{dmn} \rho^{\prime}$,

$$
\rho(A)=\sigma\left\{\rho^{\prime}(A): \rho^{\prime} \in \mathcal{K} \text { and } A \in \mathrm{dmn} \rho^{\prime}\right\},
$$

then $\rho$ is such a density that for each $\rho^{\prime} \in \mathcal{K}, \rho^{\prime}<<\rho$.

3. Definition. $\lambda$ is a lifting on $B$ if and only if $\lambda$ is a density on $B$ and for each $B \in \mathfrak{B}, \sim B \in \mathfrak{B}$ and $\lambda(\sim B)=\sim \lambda(B)$.

It immediately follows that

.1. a lifting is a density;

.2. the domain of a lifting is a field on $S$ included in $\pi$;

.3. the range of a lifting is a field on $S$ included in $\pi^{+}$;

.4. $\pi^{+} \supset F$ is a finite field implies that there is a lifting on Borelnil $F$ onto $F$. 
4. Definition. $\rho$ is a Borelnil function if and only if $\rho$ is a function on Borelnil ing $\rho$.

It immediately follows that

.1. the domain of a Borelnil function is a complete Borel field on $S$;

.2. $\pi^{+} \supset F$ is a finite field on $S$ implies that there is a Borelnil lifting on Borelnil $F$ onto $F$.

4. Lifting operators on $\mathfrak{B}(S)$.

1. Definition. $L$ is lifting operator on $B(S)$ if and only if $L$ is on $B(S)$ to $B(S)$ and, for each $f, g \in B(S)$,

.1. $L(f) \equiv f$,

.2. $f \equiv g$ implies $L(f)=L(g)$,

.3. $0 \leq f$ implíes $0 \leq L(f)$,

.4. $L\left(\chi_{s}\right)=\chi_{s}$,

.5. for each real $a, b, L(a \cdot f+b \cdot g)=a \cdot L(f)+b \cdot L(g)$,

.6. $L(f \cdot g)=L(f) \cdot L(g)$.

With regard to .5 and .6 we could require more generally that $L$, more than being just linear and multiplicative, be a homomorphism for operations which are continuous for the topology of the reals [5]. Plus and times are the two most important of such operations.

5. Differentiation and Vitali systems.

1. Definition. $F$ is a differentiation system if and only if $S \in F \subset \mathbb{M}^{+}$ and, for each $\alpha, \beta \in F, \alpha \cap \beta \in \sigma^{-} F$.

2. Definition. $F\{x\}=\{\alpha \in F: x \in \alpha\}$.

Note that if $F$ is a differentiation system, then $F\{x\}$ is a filter base. Limits with respect to $F\{x\}$ are taken in the sense of downward inclusion. $A$ theory of such limits can be found, among other places, in [12].

3. Definition. $H$ is an $F \cdot$ Vitali cover for $A$ if and only if $A \subset S, H \subset F$ $C \operatorname{sb} S$ and for each $x \in A$ and $\beta \in F\{x\}$ there is $\alpha \in H$ with $x \in \alpha \subset \beta$.

4. Definition. $F$ is a Vitali system if and only if

.1. $F$ is a differentiation system,

.2. for each $H$ and $A$ such that $H$ is an $F$-Vitali cover for $A$ there is a countable disjoint $H^{\prime} \subset H$ with $A \sim \sigma H^{\prime} \equiv 0$,

.3. $\left(\mu /\left(F \cup \pi^{0}\right)\right) \mid$ Borelnil $F=\mu \mid$ Borelnil $F$.

5. Remarks. If $\mu(S)<\infty, F \subset \mathbb{M}^{+}, \pi^{\prime}\left(F \cup \mathbb{M}^{0}\right) \subset \sigma^{\prime \prime}\left(F \cup \mathbb{M}^{0}\right)$ and $\{S\} \sim^{\prime}$ $F \subset \sigma^{\prime \prime}\left(F \cup \pi^{0}\right)$, then

$$
\left(\mu /\left(F \cup \mathbb{M}^{0}\right)\right) \mid \text { Borelinil } F=\mu \mid \text { Borelnil } F .
$$

This follows from slightly modified results in [15]. It is easy to check that 
if $\sigma^{\prime} R^{\prime} \subset \pi^{\prime \prime} R^{\prime}$, then $\sigma^{\prime} \pi^{\prime \prime} R^{\prime} \subset \pi^{\prime \prime} R^{\prime}$ so that if $R=\{S\} \sim^{\prime} F$ and $R^{\prime}=\{S\} \sim^{\prime}$ $\left(F \cup \pi^{0}\right)$, then $\sigma^{\prime} \pi^{\prime \prime} R^{\prime} \subset \pi^{\prime \prime} R^{\prime}$ and $\{S\} \sim^{\prime} R \subset \pi^{\prime \prime} R^{\prime}$. Now [15, Theorem 3.2], can be modified to read, in the notation of [15]: if $R_{\gamma} \subset R^{\prime \delta}$, then $R^{\gamma} \subset R^{\prime \delta}$. This is easily proved along lines given there. Applying this result to [15, Theorem 4.6] to get the corresponding modification of [15, Theorem 4.7], it is not hard to see that our result mentioned above stems from the complementary version of a corollary of this latter modification.

6. Fundamental theorems.

1. Theorem. If for each $n \in \omega$,

.1. $\mu(S)<\infty$,

.2. $\rho_{n}^{\prime}$ is a Borelnil density,

3. $\rho_{n}^{\prime}<<\rho_{(n+1)}^{\prime}$,

.4. $F_{n}^{\prime}=\pi^{\prime} \bigcup_{i \leq n} \mathrm{mg} \rho_{i}^{\prime}$, then for each $n \in \omega, F_{(n+1)}^{\prime} \supset F_{n}^{\prime}$ is a Vitali system.

2. Theorem. If

.1. $\mu(S)<\infty$,

.2. for each $n \in \omega, F_{(n+1)} \supset F_{n}$ is a Vitali system,

.3. $F^{*}=\bigcup_{n \in \omega} F_{n}$ and $R=$ Borelnil $F^{*}$,

.4. for each $B \in \mathscr{B}$,

$$
\rho(B)=\left\{x \in S: \lim _{n} \liminf _{\alpha \in F_{n}\{x\}} \mu(\alpha \cap B) / \mu(\alpha)=1\right\},
$$

then $\rho$ is such a Borelnil density on $B$ that for each $A \in \sigma^{-} F^{*}, A \subset \rho(A)$.

3. Corollary. If

.1. $\mu(S)<\infty$,

.2. $F^{*}$ is a Vitali system and $\mathcal{B}=$ Borelnil $F^{*}$,

.3. for each $B \in \mathbb{B}$,

$$
\rho(B)=\left\{x \in S: \lim _{a \in F^{*}\{x\}} \mu(\alpha \cap B) / \mu(\alpha)=1\right\},
$$

then $\rho$ is such a Borelnil density function on $B$ that, for each $A \in \sigma^{-} F^{*}$, $A \subset \rho(A)$.

4. Remark. 6.2 .2 is satisfied if $F$ is a nested sequence of finite fields each included in $\mathbb{M}^{+}$.

5. Remarks. As pointed out in [16], 6.2 is essentially a differentiation theorem and 6.1 provides the crucial link between a Borelnil density and the appropriate differentiating mechanism. 
Proofs and References. 6.1 is a variant of [17, Theorem 1]. Our proof is modeled after the one given there.

6.1. Proof. Clearly $F_{(n+1)}^{\prime} \supset F_{n}^{\prime}$ is a differentiation system.

Let $A \subset S$ and $H$ be an $F_{n}^{\prime}$-Vitali cover for $A$. Let $H^{\prime}$ be a maximal disjoint subfamily of $H$. Then by $\cdot 1, H^{\prime}$ is countable. Let $\beta=\rho_{n}^{\prime}\left(\sim \sigma H^{\prime}\right)$. Then by $.2, \sim \sigma H^{\prime} \equiv \beta$ and by .3 ,

$$
\sigma H^{\prime}=\bigcup_{a \in H^{\prime}} a \subset \bigcup_{a \in H^{\prime}} \rho_{n}^{\prime}(\alpha) \subset \rho_{n}^{\prime}\left(\bigcup_{a \in H^{\prime}} \rho_{n}^{\prime}(\alpha)\right)=\rho_{n}^{\prime}\left(\sigma H^{\prime}\right),
$$

so $\beta \cap \sigma H^{\prime}=0$. There can be no $x \in A \cap \beta$; for if so, then there would be $\alpha \in H$ with $x \in a \subset \beta$ in contradiction to the maximality of $H^{\prime}$. Thus $A \cap \beta$ $=0$ and $A \sim \sigma H^{\prime} \equiv 0$.

Notice that $\mu /$ field $\left(F_{n}^{\prime} \cup \mathbb{M}^{0}\right)=\mu /\left(F_{n}^{\prime} \cup \mathbb{M}^{0}\right)$. Thus $F_{n}^{\prime} \cup \mathbb{M}^{0} \mathrm{C}$ field $\left(F_{n}^{\prime} \cup \mathbb{M}^{0}\right) \subset \mathbb{M}^{\prime} \subset \mathbb{M}$, where $\mathbb{M}^{\prime}$ is the family of $\mu /\left(F_{n}^{\prime} \cup \mathbb{M}^{0}\right)$-measurable sets, and hence Borel $\left(F_{n}^{\prime} \cup M^{0}\right) \subset M^{\prime}$. Finally since $\mu$ and $\mu /\left(F_{n}^{\prime} \cup M^{0}\right)$ agree on $F_{n}^{\prime} \cup M^{0}$ and $\operatorname{Borel}\left(F_{n}^{\prime} \cup \mathbb{M}^{0}\right) \subset M^{\prime}$, we have that $\mu$ and $\mu /\left(F_{n}^{\prime} \cup M^{0}\right)$ agree on $\operatorname{Borel}\left(F_{n}^{\prime} \cup M^{0}\right)=$ Borelnil $F_{n}^{\prime}$.

Thus $F_{n}^{\prime}$ is a Vitali system.

6.2. References. See [17, Theorems 2', 2, 3, 4]. A re-examination of the proof of $\left[17\right.$, Theorem $\left.2^{\prime}\right]$ reveals that it can be slightly modified to remain valid when the condition that $\alpha \cap \beta \in F$, for $\alpha, \beta \in F$ in [17] in the definition of a differentiation system is changed to the condition that $\alpha \cap \beta$ $\epsilon \sigma^{-} F$, for $\alpha, \beta \in F$ to conform with our definition of a differentiation system. Also, if the condition that $F_{n}$ be a partial lifting (defined in [17]) in [17, Theorem 4] is changed to a Vitali system and the part of the conclusion that $A=\rho(A)$ for $A \in F^{*}$ is changed to $A \subset \rho(A)$ for $A \in \sigma^{\prime \prime} F^{*}$, then [17, Theorem 4], so revised, is valid and $\rho$ is a Borelnil density on $\mathfrak{B}$.

We now take a cue from [5]. For $H \subset F^{*}$ let $H^{\prime}$ be such a countable subfamily of $H$ that $\mu\left(\sigma H^{\prime}\right)=\sup _{\beta_{\epsilon} \sigma^{\prime \prime} H} \mu(\beta)$. Clearly from .1 for each $a \in H$, $\alpha \sim \sigma H^{\prime} \equiv 0$ so that $\rho(\alpha) \sim \rho\left(\sigma H^{\prime}\right) \equiv 0$ and $\rho(\alpha) \subset \rho\left(\sigma H^{\prime}\right)$. Thus

$$
\sigma H^{\prime} \subset \sigma H=\bigcup_{\alpha \in H} \alpha \subset \bigcup_{\alpha \in H} \rho(\alpha) \subset \rho\left(\sigma H^{\prime}\right)
$$

and

$$
\sigma H \equiv \sigma H^{\prime} \quad \text { and } \quad \sigma H^{\prime} \in \operatorname{dmn} \rho
$$

and

$$
\sigma H \subset \rho\left(\sigma H^{\prime}\right)=\rho(\sigma H)
$$

6.3. Proof. Use a constant sequence $F_{n}=F^{*}$ in 6.2 and note that for each $B \in \mathfrak{B}$ and $x \in S$, 
8

T. J. McMINN

$$
\lim _{a \in F^{*}\{x\}} \mu(\alpha \cap B) / \mu(\alpha)=1
$$

if and only if

$$
\lim _{a \in F^{*}\{x\}} \mu(\alpha \cap B) / \mu(\alpha)=1
$$

7. Commuting densities and liftings. Our main objectives in this section are Theorems 7.8, 7.11-7.14.

1. Theorem. If

.1. $G^{\prime} \subset G$ and $t^{-1} \in G^{\prime}$ for $t \in G^{\prime}$,

.2. $\pi^{0}$ is $G^{\prime}$-invariant,

.3. for each $n \in \omega, F_{n}$ is a $G^{\prime}$-invariant differentiation system,

.4. $F^{*}=\bigcup_{n \in \omega} F_{n}$ and $B=$ Borelnil $F^{*}$,

.5. for each $B \in \mathfrak{B}, x \in S$ and $t \in G^{\prime}$,

$$
\lim _{n} \lim _{\alpha \in F_{n}}\{x\}
$$

if and only if

$$
\lim _{n} \lim _{\alpha \in F_{n}\{x\}} \mu(t \cdot(\alpha \cap B)) / \mu(t \cdot \alpha)=1,
$$

6. for each $B \in \mathfrak{B}$.

$$
\rho(B)=\left\{x \in S: \lim _{n} \lim _{a \in F_{n}\{x\}} \mu(\alpha \cap B) / \mu(\alpha)=1\right\},
$$

then $\rho$ is $G^{\prime}$-commutative.

2. Corollary. If

.1. $G^{\prime} \subset G$ and if $t^{-1} \in G^{\prime}$ for $t \in G^{\prime}$,

.2. $\mathbb{M}^{0}$ is $G^{\prime}$-invariant,

.3. $F^{*}$ is a $G^{\prime}$-invariant differentiation system,

.4. $B=$ Borelnil $F^{*}$,

.5. for each $B \in \mathscr{B}$ and $t \in G^{\prime}$,

$$
\lim _{\alpha \in F^{*}\{x\}} \mu(\alpha \cap B) / \mu(\alpha)=1
$$

if. and only if

$$
\lim _{a \in F^{*}\{x\}} \mu(t \cdot(\alpha \cap B)) / \mu(t \cdot a)=1,
$$

.6. for each $B \in \mathfrak{B}$,

$$
\rho(B)=\left\{x \in S: \lim _{a \in F^{*}\{x\}} \mu(\alpha \cap B) / \mu(\alpha)=1\right\},
$$

then $\rho$ is $G^{\prime}$-commutative. 
3. Theorem. If

.1. $G$ is countable,

.2. $\pi^{0}$ is invariant,

.3. $\rho^{\prime}$ is a density on complete set $B \subset \pi$,

.4. $B$ is invariant,

.5. for each $t \in G$ and $B \in B, \rho_{t}^{\prime}(B)=t^{-1} \cdot \rho^{\prime}(t \cdot B)$ and $\rho(B)=\bigcap_{t \in G} \rho_{t}^{\prime}(B)$, then $\rho$ is a commutative density on $B$.

4. Theorem. If

.1. $\mu(S)<\infty$,

.2. $G^{\prime} \subset G$ and $t^{-1} \in G^{\prime}$ for $t \in G$,

.3. M0 is $G^{\prime}$-invariant.

.4. for each $n \in \omega, F_{(n+1)} \supset F_{n}$ is a $G^{\prime}$-invariant Vitali system,

.5. $F^{*}=\bigcup_{n \in \omega} F_{n}$ and $\mathfrak{B}=$ Borelnil $F^{*}$,

.6. for each $B \in \mathfrak{B}, x \in S$ and $t \in G^{\prime}$,

$$
\lim _{n} \liminf _{\alpha \in F_{n}\{x\}} \mu(\alpha \cap B) / \mu(\alpha)=1
$$

if and only if

$$
\lim _{n} \lim _{\alpha \in F_{n}\{x\}} \mu(t \cdot(\alpha \cap B)) / \mu(t \cdot \alpha)=1,
$$

.7. for each $B \in B_{\text {, }}$

$$
\rho(B)=\left\{x \in S: \lim _{n} \liminf _{a \in F_{n}\{x\}} \mu(\alpha \cap B) / \mu(a)=1\right\},
$$

then $\rho$ is such a $G^{\prime}$-commutative Borelnil density on $B$ that, for each $A \epsilon$ $\sigma^{-} F^{*}, A \subset \rho(A)$.

5. Theorem. If

.1. $\mu(S)<\infty$,

.2. $G$ is countable,

.3. $\pi^{0}$ is invariant,

.4. for each $n \in \omega, F(n+1) \supset F_{n}$ is a Vitali system,

.5. $F^{*}=\bigcup_{n \in \omega} F_{n}$ and $B=$ Borelnil $F^{*}$,

.6. $F^{*}$ is invariant,

7. for each $B \in \mathfrak{B}$,

$$
\rho^{\prime}(B)=\left\{x \in S: \lim _{n} \lim _{a \in F_{n}\{x\}} \mu(\alpha \cap B) / \mu(\alpha)=1\right\},
$$

.8. for each $t \in G$ and $B \in \Re$,

$$
\rho_{t}^{\prime}(B)=t^{-1} \cdot \rho^{\prime}(t \cdot B) \text { and } \rho(B)=\bigcap_{t \in G} \rho_{t}^{\prime}(B) \text {, }
$$


then $\rho$ is such a commutative Borelnil density on $B$ that, for each $A \in \sigma^{-} F^{*}$, $A \subset \rho(A)$.

\section{Lemma. If}

.1. $\mu(S)<\infty$,

.2. $G$ is countable,

.3. $\pi^{0}$ is invariant.

.4. $D \subset S$ and for each $t \in G, t \cdot D \in M^{+}$,

.5. $\rho$ " is a commutative Borelnil density,

then there is such a finite or countably infinite sequence $\rho^{\prime}$ of Borelnil densities on $(\gamma+1)$, if $\gamma$ is finite, or on $\gamma$, otherwise, that

6. for each $n \in \gamma, \rho^{\prime \prime}=\rho_{0}^{\prime} \subset \rho_{n}^{\prime} \subset \rho_{(n+1)}^{\prime}$,

.7. $D \in \operatorname{dmn} \rho_{1}^{\prime}$,

.8. for each $n \in \omega, C \in \operatorname{dmn} \rho_{n}^{\prime}$ and $t \in G$, there is such $k \in \gamma \sim(n+1)$ that $t \cdot C \in \operatorname{dmn} \rho_{(k+1)}^{\prime}$ and $\rho_{(k+1)}^{\prime}(t \cdot C)=t \cdot \rho_{(k+1)}(C)$,

.9. for each $t \in G$ and $A \in \bigcup_{n \in \operatorname{dmn} \rho} \operatorname{rng} \rho_{n}^{\prime}$,

$$
t \cdot A \in \bigcup_{n \in \operatorname{dmn} \rho} \operatorname{rng} \rho_{n}^{\prime}
$$

7. Theorem. If

.1. $\mu(S)<\infty$,

.2. $G$ is countable,

.3. $\pi^{0}$ is invariant,

.4. for each $t \in G, t \cdot D \in \mathbb{M}^{+}$,

.5. $\rho^{\prime \prime}$ is a commutative Borelnil density,

then there is such a G-commutative Borelnil density $\rho$ that $\rho^{\prime \prime} \subset \rho$ and $D \in$ $\operatorname{dmn} \rho$.

8. Theorem. If

.1. $\mu(S)<\infty$,

.2. $G$ is countable,

.3. $M$ and $M^{0}$ are invariant,

.4. for each $n \in \omega, F_{(n+1)} \supset F_{n}$ is a Vitali system,

.5. $F^{*}=\bigcup_{n \in \omega} F_{n}$,

.6. $F^{*}$ is invariant,

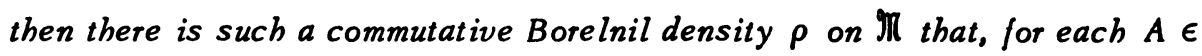
$\sigma^{-} F^{*}, A \subset \rho(A)$.

9. Lemma. If

.1. $\rho$ is a commutative dens ity on complete field $B$,

.2. $\Delta=\left\{F: F\right.$ is a field and $F \cap \cap \operatorname{rng} \rho \subset B \cap \cap \mathbb{M}^{+}$and $F$ is invariant $\}$, 
.3. $H$ is a maximal member of $\Delta$ (there is one),

.4. for each $B \in \mathfrak{B}$,

$$
\begin{aligned}
P(B)=\left\{F: \pi^{\prime}\left(\bigcup_{a \in F} \bigcup_{t \in G}\{t \cdot a\} \cup H \cup \operatorname{mg} \rho\right) \subset B \cap \pi^{+}\right. \text {and } \\
\text { for each } \alpha \in F, a \subset \sim \rho(\sim B) \text { or } a \subset \sim \rho(B)\},
\end{aligned}
$$

.5. for each $B \in \mathcal{B}, K(B)$ is a maximal member of $P(B)$ (there is one),

.6. for each $B \in \mathcal{B}, \lambda(B)=\sigma\{\alpha \in K(B): \alpha \subset \sim \rho(\sim B)\}$,

.7. for each $B \in \mathfrak{B}$ with

and

$$
\pi^{\prime}\left(\bigcup_{t \in G}\{t \cdot \lambda(B), t \cdot \lambda(\sim B)\} \cup H \cup \operatorname{rng} \rho\right) \subset \mathfrak{B} \cap \pi^{+}
$$

$$
\pi^{\prime}\left(\bigcup_{t \in G}\{t \cdot \lambda(B), t \cdot \lambda(\sim B)\} \cup H \cup \operatorname{rng} \rho\right) \sim^{\prime}\{\lambda(B), \lambda(\sim B)\} \subset B \cap \mathbb{M}^{+},
$$

for each $x \in \sim \lambda(B) \sim \lambda(\sim B), \delta \in H \cap \cap \operatorname{rng} \rho$ with $x \in \delta$ and

(a) for each nonempty finite subset $Q$ of $G$ with $0 \neq R=\{t \in Q: x=t \cdot x\}$ and $\delta \cap \bigcap_{t \in Q}(t \cdot \lambda(B))=0$ there is such $r \in G$ with $x=\tau \cdot x$ that $r \cdot \lambda(B) C$ $\bigcap_{t \in R}(t \cdot \lambda(B))$ or $r \cdot \lambda(\sim B) \subset \bigcap_{t \in R}(t \cdot \lambda(B))$ or

(b) for each nonempty finite subset $Q$ of $G$ with $0 \neq R=\{t \in Q: x=t \cdot \dot{x}\}$ and $\delta \cap \bigcap_{t \in Q}(t \cdot \lambda(\sim B))=0$ there is such $\tau \in G$ with $x=\tau \cdot x$ that $r \cdot \lambda(B)$ $\subset \bigcap_{t \in R}(t \cdot \lambda(\sim B))$ or $\tau \cdot \lambda(\sim B) \subset \bigcap_{t \in R}(t \cdot \lambda(\sim B))$,

then $\lambda$ is such a commutative lifting on $B$ onto $H$ that for each $B \in \Re, \rho(B)$ $\subset \lambda(B) \subset \sim \rho(\sim B)$.

10. Remark. If $G=\{e\}$, then for each $B \in \mathscr{B}, \lambda(B)$ reduces to

.1. $\sigma\left\{\alpha:\{\alpha\} \cap \cap H \cap \cap\right.$ rng $\rho \subset B \cap \cap \mathbb{M}^{+}$and $\left.\alpha \subset \sim \rho(\sim B)\right\}$.

In this event $\lambda(B)$ may just as well be taken to be either of the following:

.2. $\sigma\{\alpha \in H \cap \cap \operatorname{rng} \rho: \alpha \sim B \equiv 0\}$,

.3. $\bigcup_{a \in H}(\rho(a \cup B) \sim a)[17]$.

11. Theorem. If

.1. $\mu(S)<\infty$,

.2. $\mathbb{M}^{0}$ is invariant,

.3. for each $n \in \omega, F_{(n+1)} \supset F_{n}$ is an invariant Vitali system,

.4. $F^{*}=\bigcup_{n \in \omega} F_{n}$ and $\mathscr{B}=$ Borelnil $F^{*}$,

.5. for each $B \in \mathfrak{B}, x \in S$ and $t \in G$,

$$
\left.\left.\lim _{n} \lim _{a \in F} \inf _{n} \mu(\alpha)\right\} B\right) / \mu(\alpha)=1
$$

if and only if 


$$
\lim _{n} \lim _{a \in F_{n}\{x\}} \mu(t \cdot(\alpha \cap B)) / \mu(t \cdot \alpha)=1,
$$

.6. for each $a_{1}, a_{2}, \delta \in B \cap M^{+}$with $a_{1} \cap a_{2}=0, a_{1} \cup a_{2} \equiv S$,

$$
\pi^{\prime}\left(\bigcup_{t \in G}\left\{t \cdot a_{1}, t \cdot a_{2}, t \cdot \delta\right\} \cup F^{*}\right) \subset B \cap \mathbb{M}^{+}
$$

and

$$
\pi^{\prime}\left(\bigcup_{t \in G}\left\{t \cdot a_{1}, t \cdot a_{2}, t \cdot \delta\right\} \cup F^{*}\right) \sim^{\prime}\left\{a_{i}\right\} \subset B \cap \cap \pi^{+} \quad \text { for } i=1,2,
$$

for each $x \in \delta \sim a_{1} \sim \alpha_{2}$ and

(a) for each nonempty finite subset $Q$ of $G$ with $0 \neq R=\{t \in Q: x=t \cdot x\}$ and $\delta \cap \bigcap_{t \in Q}\left(t \cdot a_{1}\right)=0$ there is such $r \in G$ with $x=r \cdot x$ that $r \cdot a_{1} C$ $\bigcap_{t \in R}\left(t \cdot a_{1}\right)$ or $\tau \cdot \alpha_{2} \subset \bigcap_{t \in R}\left(t \cdot \alpha_{1}\right)$ or

(b) for each nonempty finite subset $Q$ of $G$ with $0 \neq R=\{t \in Q: x=t \cdot x\}$ and $\delta \cap \bigcap_{t \in Q}\left(t \cdot a_{2}\right)=0$ there is such $\tau \in G$ with $x=\tau \cdot x$ that $\tau \cdot a_{2} C$ $\bigcap_{t \in R}\left(t \cdot a_{2}\right)$ or $r \cdot a_{1} \subset \bigcap_{t \in R}\left(t \cdot a_{2}\right)$,

then there is such a commutatuive lifting $\lambda$ on $B$ that for each $A \in \sigma^{-} F^{*}, A \subset \rho(A)$.

12. Corollary. If 7.11.1-7.11.6 and

.7. $\mathbb{M} \subset \mathfrak{B}_{\text {, }}$

then there is such a commutative lifting $\lambda$ on $\mathbb{M}$ that, for each $A \in \sigma^{-} F^{*}, A \subset \lambda(A)$.

13. Theorem. If 7.8.1-7.8.6 and

.7. for each $a_{1}, a_{2}, \delta \in \mathbb{M}^{+}$with $a_{1} \cap a_{2}=0, a_{1} \cup a_{2} \equiv S$,

and

$$
\pi^{\prime}\left(\bigcup_{t \in G}\left\{t \cdot \alpha_{1}, t \cdot \alpha_{2}, t \cdot \delta\right\} \cup F^{*}\right) \subset \mathbb{N}^{+}
$$

$$
\pi^{\prime}\left(\bigcup_{t \in G}\left\{t \cdot a_{1}, t \cdot a_{2}, t \cdot \delta\right\} \cup F^{*}\right) \sim^{\prime}\left\{a_{i}\right\} \subset \mathbb{M}^{+} \text {for } i=1,2,
$$

$x \in \delta \sim a_{1} \sim a_{2}$ and 7.11.6((a) or (b)),

then there is such a commutative lifting $\lambda$ on $\mathbb{M}$ that, for each $A \in \sigma^{-} F^{*}$, $A \subset \rho(A)$.

14. Theorem. Consider the following conditions on $\mu$ and $G$ each inde. pendent of $F, B$ and $\rho$.

.1. for each $\alpha \in \mathbb{M}^{+}, t \in G$ and $x \in S$ with $\{x\} \in \mathbb{M}^{0}$ and $x=t \cdot x$, we have $\alpha \equiv t \cdot \alpha$;

.2. for each $t \in G$ and $x \in S$ with $\{x\} \in \mathbb{M}^{0}$ and $x=t \cdot x$, we have $t \equiv e$;

.3. for each $t \in G$ and $x \in S$ with $\{x\} \in \mathbb{M}^{0}$ and $x=t \cdot x$, we have $t=e$. Then in the context of the relevant application .3 implies .2 implies .1 implies 7.13.7 implies 7.11.6 implies 7.9.7. 
15. Theorem. If

.1. $C \in \mathbb{M}^{+}$,

.2. $G^{\prime}$ is a finite subgroup of $G$,

.3. $x \in S, r \in G^{\prime}$ and $r \cdot x=x$,

.4. $\cup_{t \in G^{\prime}}(t \cdot C) \equiv S$ and for each $t, t^{\prime} \in G^{\prime}$ with $t \neq t^{\prime},(t \cdot C) \cap(t \cdot C)$ $\equiv 0$ and $\tau \cdot t^{\prime} \cdot C \neq t \cdot C$,

then there is no G-commutative lifting on $M$.

16. Theorem. If $\lambda$ is a commutative lifting on $\mathbb{M}$ and for each $x \in S$ and $f \in \mathbb{B}(S),(L(f))(x)=\sup \left\{r: x \notin \lambda\left(f^{-1}[-\infty, r)\right)\right\}$, then $L$ is a commutative lifting operator on $\mathfrak{B}(S)$.

17. Remarks. Under the hypotheses of 7.12 or 7.13 there is a commuting lifting operator on $B(S)$. In view of 5.5, the hypotheses of 7.12 and for each $A \in F^{*}, \sim A \in \sigma^{-} F^{*}$ are satisfied when $S$ is the plane, $\mu$ is suitably normalized Lebesgue measure on $S$ and for each $n \in \omega, F_{n}$ is the family of all leftbottom-closed-right-top-open squares in $S$ and $G$ is the group of translations on $S$. In this case $F^{*} \subset$ rng $\lambda$. If for each $n \in \omega, F_{n}$ is the family of all open disks, then the hypotheses of 7.12 are satisfied and for each open $A, A \subset$ $\lambda(A)$. Similarly for Euclidean $K$-space.

18. Remark. If $\phi$ is such a Carathéodory measure on $S$ that there is a partition $\mathcal{P}$ (not necessarily countable) of $S$ into $\phi$ measurable subsets of finite $\phi$ measure with the property that, for each $A \subset S, \phi(A)=\Sigma_{B \in \mathcal{P}} \phi(A \cap B)$ and for each $B \in \mathcal{P}$ and $t \in G, t \cdot B \in \mathcal{P}$ and the relativization of $F$ to $B$ (where applicable) is a Vitali system, then each theorem in this section remains valid when ' $\mu$ ' is replaced by ' $\phi$ ', and the restriction ' $\mu(S)<\infty$ ' is deleted.

Proofs and References.

7.1. Proof. In view of .2 and $.3, B$ is invariant. Also using this fact, .1 and .5 we have, for each $B \in \mathcal{B}$ and $t \in G$,

$$
\begin{aligned}
\rho(t \cdot B) & =\left\{x \in S: \lim _{n} \liminf _{a \in F_{n}\{x\}} \mu(a \cap(t \cdot B)) / \mu(\alpha)=1\right\} \\
& =\left\{x \in S: \lim _{n} \liminf _{a \in F_{n}\left\{t^{-1} \cdot x\right\}} \mu(t \cdot(a \cap B)) / \mu(t \cdot a)=1\right\} \\
& =\left\{x \in S: \lim _{n \in F_{n}\left\{t^{-1} \cdot x\right\}} \liminf \mu(a \cap B) / \mu(a)=1\right\} \\
& =t \cdot\left\{x \in S: \lim _{a \in F_{n}\{x\}} \liminf _{n} \mu(a \cap B) / \mu(a)=1\right\} \\
& =t \cdot \rho(B) . \quad
\end{aligned}
$$


7.2. Proof. See the proof of 6.3 .

7.3. Proof. Trivially $\rho(S)=S$. Clearly from .1-.4 for each $A, B \in \mathfrak{B}$, $A \equiv \rho^{\prime}(A) \equiv \rho(A)$ and $A \equiv B$ implies $\rho(A)=\rho(B)$. To show that $\rho$ is intersective, let $A, B \in \mathfrak{B}$. Then using .3,

$$
\begin{aligned}
\rho(A \cap B) & =\bigcap_{t \in G} \rho_{t}^{\prime}(A \cap B) \\
& =\bigcap_{t \in G} t^{-1} \cdot \rho^{\prime}(t \cdot(A \cap B)) \\
& =\bigcap_{t \in G} t^{-1} \cdot\left(\rho^{\prime}(t \cdot A) \cap \rho^{\prime}(t \cdot B)\right) \\
& =\bigcap_{t \in G}\left(\left(t^{-1} \cdot \rho^{\prime}(t \cdot A)\right) \cap\left(t^{-1} \cdot \rho^{\prime}(t \cdot B)\right)\right) \\
& =\bigcap_{t \in G}\left(\rho_{t}^{\prime}(A) \cap \rho_{t}^{\prime}(B)\right) \\
& =\left(\bigcap_{t \in G} \rho_{t}^{\prime}(A)\right) \cap\left(\bigcap_{t \in G} \rho_{t}^{\prime}(B)\right)=\rho(A) \cap \rho(B) .
\end{aligned}
$$

$r$ inaliy, using the fact that $G$ is a group, for each $B \in \mathscr{B}$ and $t \in G$,

$$
\begin{aligned}
\rho(t \cdot B) & =\bigcap_{s \in G} \rho_{s}^{\prime}(t \cdot B)=\bigcap_{s \in G}\left(s^{-1} \cdot \rho^{\prime}(s \cdot t \cdot B)\right) \\
& =t \cdot \bigcap_{s \in G}\left(t^{-1} \cdot s^{-1} \cdot \rho^{\prime}(s \cdot t \cdot B)\right)=t \cdot \bigcap_{s \in G}\left((s \cdot t)^{-1} \cdot \rho^{\prime}(s \cdot t \cdot B)\right) \\
& =t \bigcap_{s \in G} \rho_{(s \cdot t)}^{\prime}(B)=t \cdot \rho(B)
\end{aligned}
$$

7.4. Proof. Use 6.2 and 7.1.

7.5. Proof. Use .3 and .6 to check that $B$ is invariant. Now use 6.2 and 7.3. Check that for each $A \in \sigma^{-F^{*}}$ and $t \in G, A \subset \rho^{\prime}(A), A \subset \rho_{t}^{\prime}(A)$ and $A \subset \rho(A)$.

Our proof of 7.6 is somewhat like that of the lemma in [17].

76 Proof. If $D \in \mathrm{dmn} \rho^{\prime \prime}$, then for each $n \leq \gamma$, if $\gamma$ is finite, or for each $n \in \gamma$, otherwise, let $\rho_{n}^{\prime}=\rho^{\prime \prime}$. Otherwise proceed as follows: Let

$$
\begin{aligned}
D^{\prime}= & D \sim \sigma\{(t \cdot D) \cap D: t \in G \text { and }(t \cdot D) \cap D \equiv 0\} \\
& \cap \sim \sigma\{(t \cdot \sim D) \cap D: t \in G \text { and }(t \cdot \sim D) \cap D \equiv 0\}, \\
D^{\prime \prime}= & \sim D \sim \sigma\{(t \cdot D) \sim D: t \in G \text { and }(t \cdot D) \sim D \equiv 0\} \\
& \cap \sim \sigma\{(t \cdot \sim D) \sim D: t \in G \text { and }(t \cdot \sim D) \sim D \equiv 0\}, \\
F^{\prime}= & \left\{a \in \operatorname{rng} \rho^{\prime \prime}: \alpha \sim D^{\prime} \equiv 0\right\} \\
F^{\prime \prime}= & \left\{a \in \operatorname{rng} \rho^{\prime \prime}: a \sim D^{\prime \prime} \equiv 0\right\}
\end{aligned}
$$


Then choose $B^{\prime} \in \sigma^{\prime \prime} F^{\prime}$ and $B^{\prime \prime} \epsilon \sigma^{\prime \prime} F^{\prime \prime}$ with

$$
\mu\left(B^{\prime}\right)=\sup _{\alpha \in \sigma^{\prime \prime} F^{\prime}} \mu(\alpha), \quad \mu\left(B^{\prime \prime}\right)=\sup _{\alpha \in \sigma^{\prime \prime} F^{\prime \prime}} \mu(\alpha) .
$$

From .1-.5 it follows that $\sigma F^{\prime} \subset \rho^{\prime \prime}\left(B^{\prime}\right) \in F^{\prime}$ and $\sigma F^{\prime \prime} \subset \rho^{\prime \prime}\left(B^{\prime \prime}\right) \in F^{\prime \prime}$, so that $\sigma F^{\prime}=\rho^{\prime \prime}\left(B^{\prime}\right)$ and $\sigma F^{\prime \prime}=\rho^{\prime \prime}\left(B^{\prime \prime}\right)$. Now let $D^{\prime \prime \prime}=D \sim \sigma^{\prime \prime}\left(B^{\prime \prime}\right)$ and $D^{\prime \prime \prime}=D^{\prime \prime} \sim \rho^{\prime \prime}\left(B^{\prime}\right)$. By .2-.4, for each $t, s \in G$,

$$
\begin{gathered}
D \equiv D^{\prime} \equiv D^{\prime \prime \prime}, \sim D \equiv D^{\prime \prime} \equiv D^{\prime \prime \prime \prime}, \quad D^{\prime \prime \prime} \cap D^{\prime \prime \prime}=0, \\
\left(t \cdot D^{\prime}\right) \cap\left(s \cdot D^{\prime}\right) \in \mathbb{M}^{+},\left(t \cdot D^{\prime}\right) \cap\left(s \cdot D^{\prime \prime}\right) \in \mathbb{M}^{+}, \\
\left(t \cdot D^{\prime \prime}\right) \cap\left(s \cdot D^{\prime \prime}\right) \in \mathbb{M}^{+},\left(t \cdot D^{\prime \prime \prime}\right) \cap\left(s \cdot D^{\prime \prime \prime}\right) \in \mathbb{M}^{+}, \\
\left(t \cdot D^{\prime \prime \prime}\right) \cap\left(s \cdot D^{\prime \prime \prime \prime}\right) \in \mathbb{M}^{+},\left(t \cdot D^{\prime \prime \prime \prime}\right) \cap\left(s \cdot D^{\prime \prime \prime \prime}\right) \in \mathbb{M}^{+}, \\
\left\{t \cdot D^{\prime \prime \prime}\right\} \cap \cap \mathrm{rng} \rho^{\prime \prime} \subset \mathbb{M}^{+}, \quad\left\{t \cdot D^{\prime \prime \prime \prime}\right\} \cap \cap \mathrm{rng} \rho^{\prime \prime} \subset \mathbb{M}^{+} .
\end{gathered}
$$

Recursively define $\rho^{\prime}$ on $(\gamma+1)$, if $\gamma$ is finite, or on $\gamma$, otherwise, so that

$$
\rho_{0}^{\prime}=\rho^{\prime \prime} \text {, }
$$

$$
\begin{aligned}
& \rho_{(n+1)}^{\prime} \text { is on field }\left(\left\{g_{n} \cdot D\right\} \cap \operatorname{dmn} \rho_{n}^{\prime}\right) \text { and } \\
& \qquad \rho_{n}^{\prime} \subset \rho_{(n+1)}^{\prime},
\end{aligned}
$$

for each $C^{\prime} \equiv g_{n} \cdot D$ and $C^{\prime \prime} \equiv g_{n} \cdot \sim D$ and $\beta, \beta^{\prime}, \beta^{\prime \prime} \in \operatorname{dmn} \rho_{n}^{\prime}$,

$$
\begin{aligned}
& \beta \cup\left(\beta^{\prime} \cap C^{\prime}\right) \cup\left(\beta^{\prime \prime} \cap C^{\prime \prime}\right) \in \operatorname{dmn} \rho_{(n+1)}^{\prime} \text { and } \\
& \rho_{(n+1)}^{\prime}\left(\beta \cup\left(\beta^{\prime} \cap C^{\prime}\right) \cup\left(\beta^{\prime \prime} \cap C^{\prime \prime}\right)\right) \\
& \quad=\rho_{n}^{\prime}(\beta) \cup\left(\rho_{n}^{\prime}\left(\beta^{\prime}\right) \cap g_{n} \cdot D^{\prime \prime \prime}\right) \cup\left(\rho_{n}^{\prime}\left(\beta^{\prime \prime}\right) \cap g_{n} \cdot D^{\prime \prime \prime \prime}\right) .
\end{aligned}
$$

Using (1) and (3) one can check by induction on $n$ that, for each $n \in \operatorname{dmn} \rho^{\prime}$, $\rho_{n}^{\prime}$ is a Borelnil density. Clearly by (2) we have .6 and by (3) we have .7 . Now given $n, m \in \gamma$, let $k \in \gamma \sim(n+1)$ so that, for each $i \leq n, g_{m} \cdot g_{i} \epsilon$ rng $(g \mid(k+1))$. With a little effort one can now use (2) and (3) to check that for each $i \leq n$ and each $C \in \operatorname{dmn} \rho_{(i+1)}^{\prime}, g_{m} \cdot C \in \operatorname{dmn} \rho_{(k+1)}^{\prime}$ and $\rho_{(k+1)}^{\prime}\left(g_{m} \cdot B\right)$ $=g_{m} \cdot \rho_{(k+1)}^{\prime}(B)$. Thus we have .8. Finally .9 follows immediately from .8 .

7.7. Proof. Use 7.6, 6.1 and 7.5.

Our proof of 7.8 is patterned after the proof of the lifting theorem in [17].

7.8. Proof. Use a maximal principle and 7.5 to get a nonempty family $K$ of such commutative Borelnil densities $\rho^{\prime \prime}$ that, for each $A \in \sigma^{-} F^{*}, A \subset \rho^{\prime \prime}(A)$, totally ordered and maximal with respect to $\ll$. Now with respect to $\ll, K$ has no cofinal countably infinite sequence $\rho^{\prime}$. For if so, then letting for each $n \in \omega, F_{n}^{\prime}=\pi^{\prime} \bigcup_{i \leq n}$ rng $\rho_{n}^{\prime}, F^{\prime *}=\bigcup_{n \in \omega} F_{n}^{\prime}$, and $B^{\prime}=$ Borelnil $F^{\prime *}$, we see that 
$F^{\prime *}$ is invariant and from 6.1 that $F_{(n+1)}^{\prime} \supset F_{n}^{\prime}$ is a Vitali system. Hence applying 7.5, we get such a commutative Borelnil density $\rho$ on $\mathfrak{B}^{\prime}$ that for each $A^{\prime} \in \sigma^{-} F^{\prime *}, A^{\prime} \subset \rho\left(A^{\prime}\right)$. It then follows that, for each $n \in \omega$ and $A \in \operatorname{dmn} \rho_{n}^{\prime}$, $\operatorname{dmn} \rho_{n}^{\prime}=$ Borelnil rng $\rho_{n}^{\prime} \subset$ Borelnil $F_{n}^{\prime} \subset$ Borelnil $F^{* *}=\mathscr{B}^{\prime}=\operatorname{dmn} \rho$,

$$
\begin{gathered}
A \in \operatorname{dmn} \rho=\mathscr{B}^{\prime} \quad \text { and } \rho_{n}^{\prime}(A) \in F_{n}^{\prime} \subset F^{\prime *} \subset \sigma^{-} F^{\prime *}, \\
\rho_{n}^{\prime}(A) \subset \rho\left(\rho_{n}^{\prime}(A)\right)=\rho(A), \quad \rho_{n}^{\prime} \ll \rho .
\end{gathered}
$$

Furthermore for each $A \in \sigma^{-} F^{\prime}, A \subset \rho_{0}^{\prime}(A) \subset \rho(A)$. Thus $\rho \in \mathcal{K}$, contradicting the cofinality of $\rho^{\prime}$.

Now for each $B \in \cup_{\rho^{\prime \prime}}{ }_{\epsilon} \mathrm{dmn} \rho^{\prime \prime}$ let

$$
\rho(B)=\sigma\left\{\rho^{\prime \prime}(B): \rho^{\prime \prime} \in \mathcal{K} \text { and } B \in \mathrm{dmn} \rho^{\prime \prime}\right\} \text {. }
$$

Then $\rho$ is such a density that, for each $\rho^{\prime \prime} \in \mathcal{K}, \rho^{\prime \prime} \ll \rho$. Clearly for each $A \epsilon$ $\sigma^{-} F^{*}, A \subset \rho(A)$. Also for each $B \in \operatorname{dmn} \rho$ and $t \in G$,

$$
\begin{aligned}
\rho(t \cdot B) & =\sigma\left\{\rho^{\prime \prime}(t \cdot B): \rho^{\prime \prime} \in \mathcal{K} \text { and } t \cdot B \in \operatorname{dmn} \rho^{\prime \prime}\right\} \\
& =t \cdot \sigma\left\{\rho(B): \rho^{\prime \prime} \in \mathcal{K} \text { and } B \in \operatorname{dmn} \rho^{\prime \prime}\right\}=t \cdot \rho(B)
\end{aligned}
$$

so that $\rho$ is commutative. Furthermore

$$
\text { Borelnil rng } \begin{aligned}
\rho & =\text { Borelnil } \bigcup_{\rho^{\prime \prime} \in K} \mathrm{rng} \rho^{\prime \prime}=\bigcup_{\rho^{\prime \prime} \in K} \text { Borelnil rng } \rho^{\prime \prime} \\
& =\bigcup_{\rho^{\prime \prime} \in K} \operatorname{dmn} \rho^{\prime \prime}=\operatorname{dmn} \rho
\end{aligned}
$$

so that $\rho$ is a Borelnil function. Thus $\rho \in \mathcal{K}$. Since $\mathcal{K}$ is maximal with respect to $\ll, \rho$ is a maximal member of $\mathcal{K}$. By .3 and 7.7 we infer from the maximality of $\rho$ in $\mathcal{K}$ that $\rho$ is on $\mathbb{N}$.

7.9. Proof. Note that $\{0, S\} \in \Delta$. Check that the union of a nest of members of $\Delta$ is a member of $\Delta$ and hence by a maximal principle that $\Delta$ has a maximal member $H$. Thus $H$ is a field, $H \cap \cap$ rng $\rho \subset B \cap \pi^{+}$and $H$ is invariant. Now let $B \in \mathbb{B}$ and note that $\{\rho(B), \rho(\sim B)\} \in P(B)$. Check that the union of a nest of members of $P(B)$ each including $\{\rho(B), \rho(\sim B)\}$ is a member of $P(B)$ including $\{\rho(B), \rho(\sim B)\}$ and hence by a maximal principle that $P(B)$ has a maximal member $K(B)$ including $\{\rho(B), \rho(\sim B)\}$. Thus

$$
\pi^{\prime}\left(\bigcup_{a \in K(B)} \bigcup_{t \in G}\{t \cdot a\} \cup H \cup \operatorname{mg} \rho\right) \subset B \cap \mathbb{M}^{+}
$$

and, for each $a \in K(B), a \subset \sim \rho(\sim B)$ or $a \subset \sim \rho(B)$. Clearly $P(B)=P(\sim B)$ and $K(B)$ is a maximal member of $P(\sim B)$ so that $\lambda(\sim B)=\sigma\{\alpha \in K(B): \alpha \subset \sim \rho(B)\}$. Also clearly $\rho(B) \subset \lambda(B) \subset \sim \rho(\sim B), \rho(\sim B) \subset \lambda(\sim B) \subset \sim \rho(B), \lambda(B) \cap \lambda(\sim B)$ $=0, \lambda(B) \equiv B$ and $\lambda(\sim B) \equiv \sim B$. Furthermore 


$$
\pi^{\prime}\left(\bigcup_{t \in G}\{t \cdot \lambda(B), t \cdot \lambda(\sim B)\} \cup H \cup \operatorname{mg} \rho\right) \subset B \cap \mathbb{M}^{+} \text {. }
$$

Now

$$
\pi^{\prime}\left(\bigcup_{t \in G}\{t \cdot \lambda(B), t \cdot \lambda(\sim B)\} \cup H \cup \operatorname{rng} \rho\right) \sim^{\prime}\{\lambda(\sim B)\} \subset \Re \cap \mathbb{M}^{+} ;
$$

for otherwise, there is

$$
\beta \in \pi^{\prime}\left(\bigcup_{t \in G}\{t \cdot \lambda(B), t \cdot \lambda(\sim B)\} \cup H \cup \operatorname{mg} \rho\right)
$$

with $0 \neq \beta \sim \lambda(\sim B) \equiv 0$ and $K(B) \cup\{\lambda(\sim B) \cup \beta\} \neq K(B) \subset K(B) \cup\{\lambda(\sim B) \cup \beta\}$ $\epsilon P(B)$ in contradiction to the maximality of $K(B) \in P(B)$. Similarly

$$
\pi^{\prime}\left(\bigcup_{t \in G}\{t \cdot \lambda(B), t \cdot \lambda(\sim B)\} \cup H \cup \operatorname{rng} \rho\right) \sim^{\prime}\{\lambda(B)\} \subset B \cap \pi^{+} .
$$

To be sure that $\lambda$ has all of the other desired properties, we need only show, since $H \subset \mathcal{B}$, that $\lambda(B) \in H$ or $\lambda(\sim B) \in H$. We may suppose that $\lambda(B) \neq 0$ and $\lambda(B) \neq S$. To this end if $(a)$, then let

and if (b), then let

$$
H^{\prime}=\text { field }\left(H \cup \bigcup_{t \in G}\{t \cdot \lambda(B)\}\right)
$$

$$
H^{\prime}=\text { field }\left(H \cup \bigcup_{t \in G}\{t \cdot \lambda(\sim B)\}\right) \text {. }
$$

We may suppose (a), and attempt to show that $\lambda(B) \in H$, since the alternate assumption entails a quite symmetric argument leading to $\lambda(\sim B) \in H$. Clearly $H^{\prime}$ is an invariant field. Since $B$ is complete, $\lambda(B) \in \mathscr{B}$ and rng $\rho \subset \mathcal{B}$. Since $H \subset \mathbb{B}$ and $\mathscr{B} \supset$ rng $\rho$ is invariant, $H^{\prime} \subset \mathcal{B}$ and $H^{\prime} \cap \cap$ rng $\rho \subset \mathcal{B}$. We shall show that $H^{\prime} \in \Delta$ by showing that $H^{\prime} \cap \cap \mathrm{mg} \rho \subset \mathbb{M}^{+}$. To do this we let $a \in H^{\prime}$ and $b \in \operatorname{mg} \rho$ and then show that $a \cap b \in \mathbb{M}^{+}$. Thus let

$$
\begin{aligned}
a= & \eta \cup\left(\xi_{0} \cap \bigcap_{t \in F_{0}}(t \cdot \lambda(B)) \cap \bigcap_{t \in F_{0}^{\prime}}(t \cdot \sim \lambda(B))\right) \cup \ldots \\
& \cup\left(\xi_{p} \cap \bigcap_{t \in F_{p}}(t \cdot \lambda(B)) \cap \bigcap_{t \in F_{p}^{\prime}}(t \cdot \sim \lambda(B))\right),
\end{aligned}
$$

where $p \in \omega, \eta, \xi_{0}, \ldots, \xi_{p} \in H, F_{0}, F_{0}^{\prime}, \ldots, F_{p}, F_{p}^{\prime}$ are finite subsets of $G$. Let us suppose, contrary to what we wish to show, that

$$
0 \neq a \cap b \equiv 0 \text {. }
$$

Since clearly $\eta \cap b=0$, by virtue of $H \cap \cap \operatorname{rng} \rho \subset \mathbb{M}^{+}$, we may suppose that there is $n \leq p$ and $x^{\prime} \in S$ with 


$$
x^{\prime} \in b \cap \xi_{n} \cap \bigcap_{t \in F_{n}}(t \cdot \lambda(B)) \cap \bigcap_{t \in F_{n}^{\prime}}(t \cdot \sim \lambda(B)) \equiv 0 .
$$

If $F_{n}^{\prime}=0$, then (noticing that $\bigcap_{t \in 0}(t \cdot \sim \lambda(B))=S$ ) by virtue of the fact that $\lambda(B) \subset \sigma K(B)$, there is such a function $k$ on $F_{n}$ to $K(B)$ that, by virtue of (1),

$$
\begin{gathered}
x^{\prime} \in b \cap \xi_{n} \cap \bigcap_{t \in F_{n}}(t \cdot k(t)) \subset b \cap \beta_{n} \cap \bigcap_{t \in F_{n}}(t \cdot \lambda(B)) \equiv 0, \\
b \cap \xi_{n} \cap \bigcap_{t \in F_{n}}(t \cdot k(t)) \in \pi^{\prime}\left(\bigcup_{a \in K(B)} \bigcup_{t \in G}\{t \cdot a\} \cup H \cup \text { rng } \rho\right) \subset \pi^{+},
\end{gathered}
$$

a contradiction. Thus $F_{n}^{\prime} \neq 0$ and there is such a

$$
\zeta^{\prime} \in \pi^{\prime}\left(\bigcup_{\alpha \in K(B)} \bigcup_{t \in G}\{t \cdot a\} \cup H \cup \operatorname{ring} \rho\right)
$$

that $x^{\prime} \in \zeta^{\prime} \cap \bigcap_{t \in F_{n}^{\prime}}(t \cdot \sim \lambda(B)) \equiv 0$. But then, since for each $t \in G, t \cdot$ $\lambda(\sim B) \subset t \cdot \sim \lambda(B)$, we infer that $\zeta^{\prime} \cap \bigcap_{t \in F_{n}^{\prime}}(t \cdot \lambda(\sim B)) \subset \zeta^{\prime} \cap \bigcap_{t \in F_{n}^{\prime}}(t \cdot \sim \lambda(B))$ $\equiv 0$. Since $x^{\prime} \in \zeta^{\prime}$ and, from $(1), \zeta^{\prime} \cap \bigcap_{t \in F_{n}^{\prime}}(t \cdot \lambda(\sim B))=0$ we have $x^{\prime} \notin$ $\bigcap_{t \in F_{n}^{\prime}}(t \cdot \lambda(\sim B))$, and hence

$$
\begin{aligned}
x^{\prime} & \in \zeta^{\prime} \cap \bigcap_{t \in F_{n}^{\prime}}(t \cdot \sim \lambda(B)) \sim \bigcap_{t \in F_{n}^{\prime}}(t \cdot \lambda(\sim B)) \\
& =\zeta^{\prime} \cap \bigcup_{t \in F_{n}^{\prime}}(t \cdot \sim \lambda(\sim B)) \cap \bigcap_{t \in F_{n}^{\prime}}(t \cdot \sim \lambda(B)) \\
& =\zeta^{\prime} \cap \bigcup_{t^{\prime} \in F_{n}^{\prime}}\left(\left(t^{\prime} \sim \lambda(\sim B) \cap \bigcap_{t \in F_{n}^{\prime}}(t \cdot \sim \lambda(B))\right)\right. \\
& \subset \zeta^{\prime} \cap \bigcup_{t^{\prime} \in F_{n}^{\prime}}\left(\left(t^{\prime} \cdot \sim \lambda(\sim B)\right) \cap(t \cdot \sim \lambda(B))\right) .
\end{aligned}
$$

Thus for some $t^{\prime} \in F_{n}^{\prime}, x^{\prime} \in \zeta^{\prime} \cap\left(t^{\prime} \cdot \sim \lambda(\sim B)\right) \cap\left(t^{\prime} \cdot \sim \lambda(B)\right)$. Letting $x=$ $t^{\prime-1} \cdot x^{\prime}$ and $\zeta=t^{\prime-1} \cdot \zeta^{\prime}$ we have

(5) $x \in \zeta \sim \lambda(\sim B) \sim \lambda(B)$ and $\zeta \in \pi^{\prime}\left(\bigcup_{a \in K(B)} \bigcup_{t \in G}\{t \cdot a\} \cup H \cup \operatorname{rng} \rho\right)$.

Now let $B^{\prime}=\lambda(B) \cup\{x\}$ and let $K^{\prime}(B)=K(B) \cup\left\{B^{\prime}\right\}$. We shall show that $K^{\prime}(B) \in P(B)$ by showing that

and

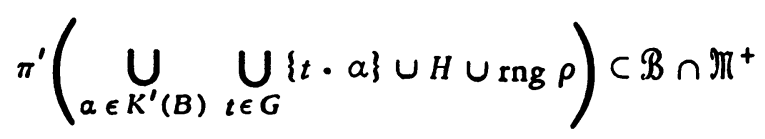

$$
\text { for each } \alpha \in K^{\prime}(B), \quad \alpha \subset \sim \rho(\sim B) \text { or } a \subset \sim \rho(B) \text {. }
$$

To this end note, since $\lambda(B) \subset \sim \lambda(\sim B)$ and $x \in \sim \lambda(\sim B)$, that $B^{\prime} \subset \sim \lambda(\sim B)$ so that (6) and suppose, contrary to what we have left to show, that there are such $\delta^{\prime} \in H \cap \cap \mathrm{rng} \rho$ and a nonempty finite set $Q \subset G$ that $0 \neq \delta \cap \bigcap_{t \in Q^{\left(t \cdot B^{\prime}\right)}}$ 
$\equiv 0$. But then $\delta \cap \bigcap_{t \in G}(t \cdot \lambda(B)) \equiv 0$. By (1), $\delta \cap \bigcap_{t \in G}(t \cdot \lambda(B))=0$ and hence $\delta \cap^{\prime} \bigcap_{t \in Q}\left(t \cdot B^{\prime}\right)=\{x\}$. Thus $x \in \delta$ and for each $t \in Q, x=t \cdot x$ or $x \in t$. $\lambda(B)$. Thus letting $R=\{t \in Q: x=t \cdot x\}$, we have $x \in \delta \cap \bigcap_{t \in Q \sim R}(t \cdot \lambda(B))$. If $R=0$, then from (5) and (1) we get the contradiction $0 \equiv\{x\}=\delta \cap$ $\bigcap_{t \in Q}\left(t \cdot B^{\prime}\right)=\delta \cap \bigcap_{t \in Q}(t \cdot \lambda(B)) \in \mathbb{M}^{+}$. So $R \neq 0$ and, upon glancing at (1), (2) and (3), we infer from .7 that there is such $r \in G$ with $x=\tau \cdot x$ that $r \cdot$ $\lambda(B) \subset \bigcap_{t \in R}(t \cdot \lambda(B))$ or $\tau \cdot \lambda(\sim B) \subset \bigcap_{t \in R}(t \cdot \lambda(B))$. Clearly then $(\tau \cdot \lambda(B))$ $\cap \delta \cap \bigcap_{t \in Q \sim R}(t \cdot \lambda(B))=0$ or $\left.\tau \cdot \lambda(\sim B)\right) \cap \delta \cap \bigcap_{t \in Q \sim R}(t \cdot \lambda(B))=0$ and hence

or

$$
\lambda(B) \cap\left(\tau^{-1} \cdot \delta\right) \cap \bigcap_{t \in Q \sim R}\left(\tau^{-1} \cdot t \cdot \lambda(B)\right)=0
$$

But also

$$
\lambda(\sim B) \cap\left(\tau^{-1} \cdot \delta\right) \cap \bigcap_{t \in Q \sim R}\left(\tau^{-1} \cdot t \cdot \lambda(B)\right)=0 .
$$

so that from (5)

$$
x=\tau^{-1} \cdot x \in\left(\tau^{-1} \cdot \delta\right) \cap \bigcap_{t \in Q}\left(\tau^{-1} \cdot t \cdot \lambda(B)\right)
$$

or

$$
\left.x \in\left(\tau^{-1} \cdot \delta\right) \cap \bigcap_{t \in Q \sim R}\left(\tau^{-1} \cdot t \cdot \lambda(B)\right) \sim \lambda(\sim B)\right) \equiv 0
$$

$$
x \in\left(\tau^{-1} \cdot \delta\right) \cap \bigcap_{t \in Q \sim R}\left(\tau^{-1} \cdot t \cdot \lambda(B)\right) \sim \lambda(B) \equiv 0
$$

in contradiction to (2) or (3). Thus

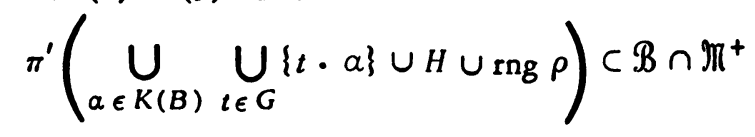

and $K(B) \subset K^{\prime}(B) \in P(B)$. Now since $K(B)$ is maximal in $P(B)$, we have $B^{\prime} \epsilon$ $K^{\prime}(B)=K(B)$ and $x \in B^{\prime} \subset \sigma K^{\prime}(B)=\sigma K(B)=\lambda(B)$ in contradiction to (5). Thus our supposition (4) is false and $a \cap b \in \mathbb{M}^{+}$and $H \subset H^{\prime} \in \Delta$. Since $H$ is maximal in $\Delta$ we have finally $\lambda(B) \in H^{\prime}=H$, which is what we set out to prove.

7.11. Proof. Use 7.4 with $G=G^{\prime}$ and 7.9 , noting that 7.11 .6 implies 7.9.7.

7.12. Proof. Immediate.

7.13 Proof. Use 7.8 and 7.9 with $B=\pi$ in 7.9 , noting that $7.13 .7 \mathrm{im}$ plies 7.9.7 with $\mathfrak{B}=\mathbb{M}$.

7.14. Proof. Immediate.

7.15. Proof. Suppose there were a G-commutative lifting $\lambda$ on $M$. Then clearly for each $t, t^{\prime} \in G^{\prime}$ with $t \neq t^{\prime}, \lambda\left(t^{\prime} \cdot C\right) \cap \lambda(t \cdot C)=0$ and $\bigcup_{t \in G^{\prime}} \lambda(t \cdot C)$ $=S$. But then for some $t \in G^{\prime}, x \in \lambda(t \cdot C)$. Thus we get the contradiction $x=$ $r \cdot x \in \tau \cdot \lambda(t \cdot C)=\lambda(r \cdot t \cdot C) \neq \lambda(t \cdot C)$ and $\lambda(\tau \cdot t \cdot C) \cap \lambda(t \cdot C)=0$.

7.16. Reference. Commutativity of $L$ is immediate. The proof of the rest is given in [s]. 
9. Density topologies and strong lifting operators. Our main objectives in this section are Theorems 8.3 and 8.6.

1. Theorem. If $\mu(S)<\infty, \rho$ is a density on a complete Borel field $\mathfrak{B}$ and $T=\sigma^{-} \mathrm{rng} \rho$, then $\mathbb{B} \cap \mathbb{M}^{+} \supset T$ is a topology on $S$ and for each $\beta \in T, \beta \subset$ $\rho(\beta) \subset \operatorname{clsr}_{T}(\beta)$.

2. Theorem. If $\mu(S)<\infty, \rho$ is a density on complete Borel field $\mathscr{B}$ and $\sigma^{-} \operatorname{rng} \rho \supset \mathcal{J}$ is regular, then there is such a lifting $\lambda$ on $B$ that $\mathscr{T} \subset \sigma^{-}$rng $\lambda$.

3. Theorem. If

.1. $\mu(S)<\infty$,

.2. for each $n \in \omega, F_{(n+1)} \supset F_{n}$ is a Vitali system,

3. $F^{*}=\bigcup_{n \in \omega} F_{n}$,

.4. $\mathcal{S} \subset \sigma^{-} F^{*}$,

.5. either $\mathcal{T}$ is regular or for each $A \in F^{*}, \sim A \in \sigma^{-} F^{*}$,

then there is such a lifting $\lambda$ on $\mathbb{N}$ that $\mathcal{S} \subset \sigma^{-} \mathrm{rng} \lambda$.

4. Lemma. If $\mu(S)<\infty$ and $\pi^{+} \supset \mathcal{T}$ is metrizable, then $\mathcal{T}$ has a countable base.

5. Remark. If $\mathfrak{T}$ is regular and has a countable base, then $\mathfrak{T}$ is metrizable.

6. Theorem. If $\mu(S)<\infty$ and $\mathbb{M}^{+} \supset \mathcal{T}$ is metrizable, then there is a lifting

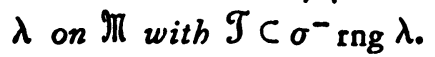

7. Definition. $L$ is $\mathfrak{T}_{\text {-strong }}$ if and only if for each $\mathfrak{T}_{\text {-continuous }} f \epsilon$ $B(s), L(f)=f$.

8. Theorem. If

.1. $\mu(S)<\infty$,

.2. $\lambda$ is a lifting on $M$,

.3. $\mathcal{S} \subset \sigma^{-} \mathrm{rng} \lambda$,

.4. for each $x \in S$ and $f \in \mathfrak{B}(S),(L(f))(x)=\sup \left\{r: x \notin \lambda\left(f^{-1}[-\infty, r)\right)\right\}$, then $L$ is a strong lifting operator on $\mathbb{B}(S)$.

9. Remark. Referring to the application of 7.12 mentioned in 7.17 , since for either choice of $F, \mathcal{J} \subset \sigma^{-} F^{*}$ and the hypotheses of 8.3 are satisfied, we infer that $\mathcal{I} \subset \sigma^{-}$rng $\lambda$ and the lifting operator $L$ associated with $\lambda$ mentioned in 8.7 is strong.

10. Remarks. If $\phi$ is as in 7.18 and in addition $\mathcal{P} \subset \mathcal{T}$, then $8.1,8.2$ and 8.6 become valid when ' $\mu$ ' is replaced by ' $\phi$ ' and the restriction ' $\mu(S)<\infty$ ' is deleted. Under this circumstance there is a strong lifting operator on $P(S)$. 


\section{Proofs and References.}

8.1. Reference. See [5], [7], [13]. The proof given in [5] is for a lifting on $\pi$ but it remains valid also for a density on a complete Borel field.

The last step in the proof of 6.2 is like one in [5] used to show that $T \subset \mathbb{M}^{+}$. That ing $\rho$ is a base for a topology follows from the fact that $\rho$ is intersective.

8.2. Reference and Proof. See [7]. For sake of completeness, we repeat the short proof. We use 7.9 with $G=\{e\}$ to get such a lifting $\lambda$ on $\mathfrak{B}$ that for each $B \in B, \rho(B) \subset \lambda(B) \subset \sim \rho(\sim B)$. Now let $x \in a \in \mathfrak{T}$. By regularity there is $\beta \in \mathfrak{T}$ with $x \in \beta \subset$ clsr $\beta \subset \alpha$. Thus from 8.1, $x \in \beta \subset \rho(\beta) \subset \lambda(\beta)$ $\subset \sim \rho(\sim \beta)$. But also $\sim \operatorname{clsr} \beta \subset \rho(\sim \operatorname{clsr} \beta) \subset \rho(\sim \beta)$. So $x \in \beta \subset \rho(\beta) \subset \lambda(\beta)$ $C \sim \rho(\sim \beta) \subset$ clsr $\beta \subset \alpha$.

8.3. Proof. Suppose $\mathcal{T}$ is regular. Use 7.13 with $G=\{e\}$ to get such a lifting $\lambda$ on $\mathbb{N}$ that if $A \in \sigma^{-} F^{*}$, then $A \subset \lambda(A)$. Now let $x \in a \in \mathcal{T}$ and use regularity to get $\beta \in \mathfrak{T}$ with $x \in \beta \subset$ clss $\beta \subset \alpha$. Thus $x \in \beta \subset \lambda(B) \subset$ $\lambda(\sim$ clsr $\beta) \subset$ clsr $\beta \subset \alpha$. In the other event the conclusion is immediate from 7.13 with $G=\{e\}$ since $F^{*} \subset \mathrm{rng} \lambda$.

8.4. Proof. For any $c \in(0 ; \infty)$ there can be at most a countable number of disjoint open spheres of radius $c$. From this fact a countable dense set can be got.

8.5. Reference. For this form of metrization theorem see [4].

8.6. Proof. If $S$ is a singleton the case is trivial. Otherwise use 8.4 to secure a sequence $b$ of open sets, each nonempty and not equal to $S$ and each equal to the interior of its closure, which form a base for $\mathcal{T}$. Note that for each $m, n \in \omega, b_{n} \not S \not \sim b_{n}$ and $b_{n} \equiv b_{m}$ implies $b_{n}=b_{m}$. Define $H$ recursively on $\omega$ so that for each $n \in \omega$,

$$
\begin{aligned}
H_{0} & =\left\{0, S, b_{0}, \sim b_{0}\right\}, \\
H_{(n+1)}=\sigma^{\prime} \pi^{\prime}\left(\left(H _ { n } \sim ^ { \prime } \left(\left(H _ { n } \cap \cap \left\{b_{(n+1)},\right.\right.\right.\right.\right. & \left.\left.\left.\left.\sim b_{(n+1)}\right\}\right) \cap \pi \pi^{0}\right)\right) \\
& \left.\cup \text { rng }(b \mid(n+2)) \cup\left\{\sim b_{(n+1)}\right\}\right) .
\end{aligned}
$$

For each $n \in \omega$ and $B^{\prime} \equiv B \in H_{n}$ let $\rho_{n}^{\prime \prime}\left(B^{\prime}\right)=\sigma\left\{\alpha \in H_{n}: \alpha \equiv B^{\prime}\right\}$. Check by induction on $n$ that for each $n \in \omega, \pi^{+} \supset \operatorname{rng} \rho_{n}^{\prime \prime}$ is finite, dmn $\rho_{n}^{\prime \prime}=$ Borelnil $H_{n},\{0, S\} \cup \mathrm{rng}(b \mid(n+1)) \subset$ rng $\rho_{n}^{\prime \prime}$ and $\rho_{n}^{\prime \prime}$ is a Borelnil density. For each $n \in \omega$ and $B \in \operatorname{dmn} \rho_{n}^{\prime \prime}$ let $\rho_{n}^{\prime}(B)=\bigcap_{m \geq n} \rho_{m}^{\prime \prime}(B)$. Check that for each $n \in \omega, \rho_{n}^{\prime}$ is a Borelnil density, $\rho_{n}^{\prime} \subset \rho_{(n+1)}^{\prime}, \rho_{n}^{\prime} \ll \rho_{(n+1)}^{\prime}$, rng $(b \mid(n+1))$

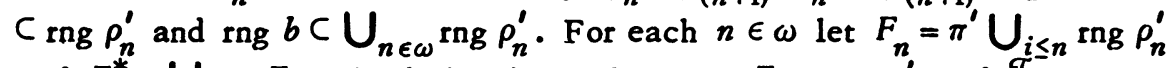
and $F^{*}=\bigcup_{n \in \omega} F_{n}$. Check that for each $n \in \omega, F_{n}=\operatorname{rng} \rho_{n}^{\prime}$ and $\mathcal{J}^{i \leq n}=$ $\sigma^{-}$rng $b \subset \sigma^{-} F^{*}$. Now apply 6.1 to see that for each $n \in \omega, F_{(n+1)} \supset F_{n}$ is a 
Vitali system. Finally, noting that $\mathfrak{T}$ is regular, use 8.3 to get such a lifting $\lambda$ on $M$ that $\mathcal{S} \subset \sigma^{-}$rng $\lambda$.

8.8. Reference. The proof is given in [5].

\section{REFERENCES}

1. K. Bichteler, On the strong lifting property, Illinois J. Math. 16 (1972), 370380. MR $45 \# 5311$.

2. - An existence theorem for strong liftings, J. Math. Anal. Appl. 33 (1971), 20-22. MR $42 \# 4700$.

3. - A reduction of the strong lifting problem, Invent. Math. 11 (1970), 159-162. MR 44 \#2906.

4. J. Dugundji, Topology, Allyn and Bacon, Boston, Mass., 1966, 9.1 Theorem (J. Nagata and Yu. M. Smirnov), pp. 193-194. MR 33 \# 1824.

5. P. A. Fillmore, On the topology induced by measure, Proc. Amer. Math. Soc. 17 (1966), 854-857. MR 33 \#5830.

6. J. Gapaillard, Relèvements sur une algèbre de parties d'un ensemble, C. R. Acad. Sci. Paris Sér. A-B 274 (1972), A1798-A1800. MR 45 \#7014.

7. A. Ionescu-Tulcea and C. Ionescu-Tulcea, Topics in the theory of lifting, Ergebnisse der Mathematik und ihrer Grenzgebiete, Band 48, Springer-Verlag, New York, 1969. MR 43 \#2185.

8. - Liftings for abstract valued functions and separable stochastic processes, Z. Wahrscheinlichkeitstheorie und Verw. Gebiete 13 (1969), 114-118. MR $43 \# 2763$.

9. C. Ionescu-Tulcea, On liftings and derivation bases, J. Math. Anal. Appl. 35 (1971), 449-466. MR 43 \#7583.

10. - Liftings for functions with values in a completely regular space, Math. Ann. 187 (1970), 200-206. MR 42 \# 1973.

11. - On the lifting property and disintegration of measures, Bull. Amer. Math. Soc. 71 (1965), 829-842. MR 34 \#600.

12. H. Kenyon and A. P. Morse, Runs, Pacific J. Math. 8 (1958), 811-824. MR $21 \# 2967$.

13. D. Maharam, On a theorem of von Neumann, Proc. Amer. Math. Soc. 9 (1958), 987-994. MR 21 \#4220.

14. R. Maher, A note on strong liftings, J. Math. Anal. Appl. 29 (1970), 633-639. MR 43 \#7584.

15. A. P. Morse, The role of internal families in measure theory, Bull. Amer. Math. Soc. 50 (1944), 723-728. MR 6, 120.

16. J. von Neumann, Algebräsche Repräsentanten der Funktionen bis auf eine Menge von Masse null, J. Reine Angew. Math. 165 (1931), 109-115.

17. M. Sion, $A$ theory of semigroup valued measures, Springer-Verlag, New York, 1973, Appendix.

18. - Introduction to the methods of real analysis, Holt, Rinehart and Winston, New York, 1968. MR 37 \#5332.

19. T. Traynor, An elementary proof of the lifting theorein, Pacific J. Math. 53 (1974), 267-272.

DEPARTMENT OF MATHEMATICS, UNIVERSITY OF NEVADA, RENO, NEVEDA 89507 